\title{
Ferroelectric Dynamics in the Perovskite Relaxor PMN
}

\author{
P. M. Gehring* ${ }^{*}$ S. Wakimoto ${ }^{\dagger}$, Z.-G. Ye ${ }^{* *}$ and G. Shirane \\ *NIST Center for Neutron Research, National Institute of Standards and Technology, Gaithersburg, \\ MD 20899-8562 \\ ${ }^{\dagger}$ Department of Physics, University of Toronto, Toronto, Ontario, Canada M5S 1 A7 \\ ${ }^{*}$ Department of Chemistry, Simon Fraser University, Burnaby, British Columbia, Canada V5A 1S6 \\ ${ }^{\ddagger}$ Department of Physics, Brookhaven National Laboratory, Upton, NY 11973-5000
}

\begin{abstract}
We review results obtained from recent neutron scattering studies of the lead-oxide class of perovskite relaxors PMN and PZN. A ferroelectric soft mode has been identified in PMN at $1100 \mathrm{~K}$ that becomes overdamped near $620 \mathrm{~K}$. This is the same temperature at which polar nanoregions (PNR) begin to form, denoted by $T_{d}$, and suggests that a direct connection exists between the soft mode and the PNR. The appearance of diffuse scattering intensity at $T_{d}$ reported by Naberezhnov et al. lends further support to this picture. At lower temperature the soft mode in PMN reappears close to $T_{c}=213 \mathrm{~K}$ (defined only for $E>E_{c}$ ). These results are provocative because the dynamics below $T_{c}$ are characteristic of an ordered ferroelectric state, yet they occur in a system that remains cubic on average at all temperatures. We discuss a coupled-mode model that successfully describes these data as well as those from earlier lattice dynamical studies of other perovskites such as $\mathrm{BaTiO}_{3}$.
\end{abstract}

\section{INTRODUCTION - SOFT MODES AND PNR}

Recent neutron scattering studies of the perovskite $\left(A B \mathrm{O}_{3}\right)$ relaxors $\mathrm{Pb}\left(\mathrm{Mg}_{1 / 3} \mathrm{Nb}_{2 / 3}\right) \mathrm{O}_{3}$ $(\mathrm{PMN})$ and $\mathrm{Pb}\left(\mathrm{Zn}_{1 / 3} \mathrm{Nb}_{2 / 3}\right) \mathrm{O}_{3}(\mathrm{PZN})$ have focused on the low-energy $(\leq 20 \mathrm{meV})$ lattice dynamics to determine whether or not a soft mode picture is relevant to these chemically-disordered systems. [1], 2, 3] The answer to this question is not obvious in spite of the similarities between PMN and PZN, and the displacive ferroelectric $\mathrm{PbTiO}_{3}$, which exhibits a phase transition to a tetragonal structure at $T_{c}=763 \mathrm{~K}$. The occupation of the $B$-site by either $\mathrm{Mg}^{2+}$ or $\mathrm{Zn}^{2+}$, and $\mathrm{Nb}^{5+}$ cations creates a local charge imbalance, and gives rise to rapidly varying random fields. [ $₫$ ] Therefore anomalies in the lattice dynamics of both PMN and PZN are to be expected. Of particular interest is the role played by the polar nanoregions (PNR), which for PMN form between 600 and $650 \mathrm{~K}$. [5] Given their polar nature they ought to couple strongly to polar phonon modes, including any soft modes if present, in the relaxors PMN and PZN.

The existence of a soft transverse optic (TO) phonon mode has been documented in numerous perovskites including $\mathrm{PbTiO}_{3}, \mathrm{BaTiO}_{3}, \mathrm{SrTiO}_{3}$, and $\mathrm{KTaO}_{3}$. In the case of $\mathrm{PbTiO}_{3}$, the frequency of the lowest-lying $\mathrm{TO}$ mode drops when cooled from high temperature and condenses at the (first-order) transition temperature $T_{c}=763 \mathrm{~K}$, transforming the system into a tetragonal state. [6] Unlike those in other perovskites, the soft mode in $\mathrm{PbTiO}_{3}$ is well defined except for a limited range of reduced wave vectors 


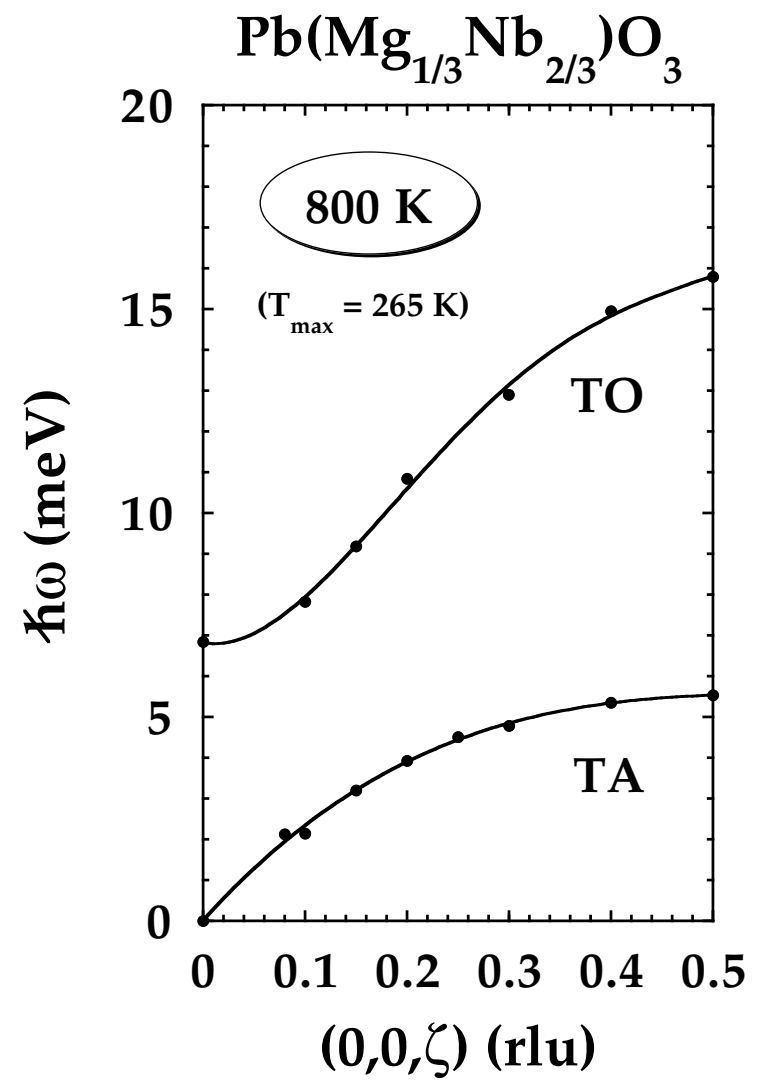

FIGURE 1. PMN dispersion measured at $800 \mathrm{~K}$ near (221). Data taken from Naberezhnov et al. 㳍

$q \leq 0.08$. The zone center $q=0$ mode is, however, rather broad and well defined only at temperatures far above $T_{c}$. [6]

Pioneering lattice dynamical studies were carried out by Naberezhnov et al. on PMN from $300 \mathrm{~K}$ to $900 \mathrm{~K}$. [7] Fig. 1 shows the TO and TA phonon dispersions for PMN measured at $800 \mathrm{~K}$ around (221). A well defined zone center TO mode is observed at this temperature. However, it was identified as a hard TO1 mode in this study because its structure factor was inconsistent with those obtained from neutron diffuse scattering measurements by Vakhrushev et al. [8] Prior Raman scattering studies had also found no evidence of a soft mode in PMN. [9]

Subsequent experiments by Gehring et al. were done on PZN and PZN doped with 8\% $\mathrm{PbTiO}_{3}(\mathrm{PZN}-8 \% \mathrm{PT})$ for which large good quality single crystals were readily available. Fig. 2 combines a series of constant $-\vec{Q}$ spectra measured on a 4.2 gm single crystal of PZN at $500 \mathrm{~K}$, nearly $100 \mathrm{~K}$ above $T_{c}$, into a greyscale contour plot (see reference for experiment details). [2] These data show an unusual feature in which the TO branch appears to drop into the TA branch around a $q$ of just under 0.15 reciprocal lattice units $\left(1 \mathrm{rlu}=1.545 \AA^{-1}\right)$, which is a measure of the average size of the PNR. This anomaly, termed "the waterfall" because of its vertical appearance, occurs as a result of a $q$-dependent damping in which the TO phonon mode becomes increasingly damped with decreasing wave vector $q$, i. e. increasing wavelength. No phonon peaks are observed in 


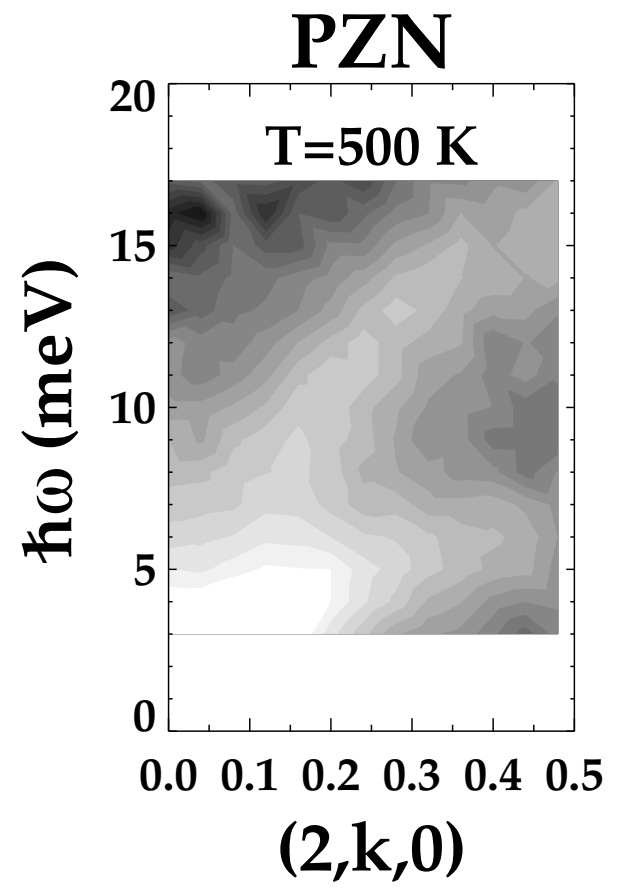

FIGURE 2. Contour plot derived from neutron inelastic constant $-\vec{Q}$ scans measured on PZN at $500 \mathrm{~K}$ near (200). From Gehring et al. [2] Lighter regions indicate higher intensity.

constant- $\vec{Q}$ scans for $q$ near the zone center, which indicates that these TO modes are overdamped. Thus the waterfall feature is not a true dispersion because the scattering does not come from propagating modes. The waterfall is seen in PMN as well as in solid solutions of both PZN and PMN and $\mathrm{PbTiO}_{3}(\mathrm{PZN}-x \mathrm{PT}$ and PMN- $x \mathrm{PT})$, suggesting it may be common to all relaxor systems. [10, 11]

In comparison to $\mathrm{PbTiO}_{3}$, these results on $\mathrm{PZN}$ reveal the presence of a new damping mechanism that effectively freezes out the longest-wavelength TO modes and produces the unusual waterfall feature. The PNR are the most plausible cause of this damping. To test this idea would require heating the PZN crystal to temperatures well in excess of $T_{d} \approx 760 \mathrm{~K}$ where the PNR are no longer present. Because this was deemed too close to the decomposition temperature of PZN, this experiment was performed on PMN, for which $T_{d} \approx 620 \mathrm{~K}$ as first determined by the optical measurements of Burns and Dacol. [5] As was the case in PZN, an overdamped response was observed in PMN at $q=0$ at $500 \mathrm{~K}$. However the data in Fig. 3 show unambiguous evidence of a well defined peak at $q=0$ at $1100 \mathrm{~K}$ that softens upon cooling. At the same time the mode becomes increasingly damped (broader energy linewidth). Within experimental uncertainty the soft mode becomes overdamped at $T_{d}$, thereby establishing a definite connection between it and the PNR. Equally interesting is the fact that the square of 


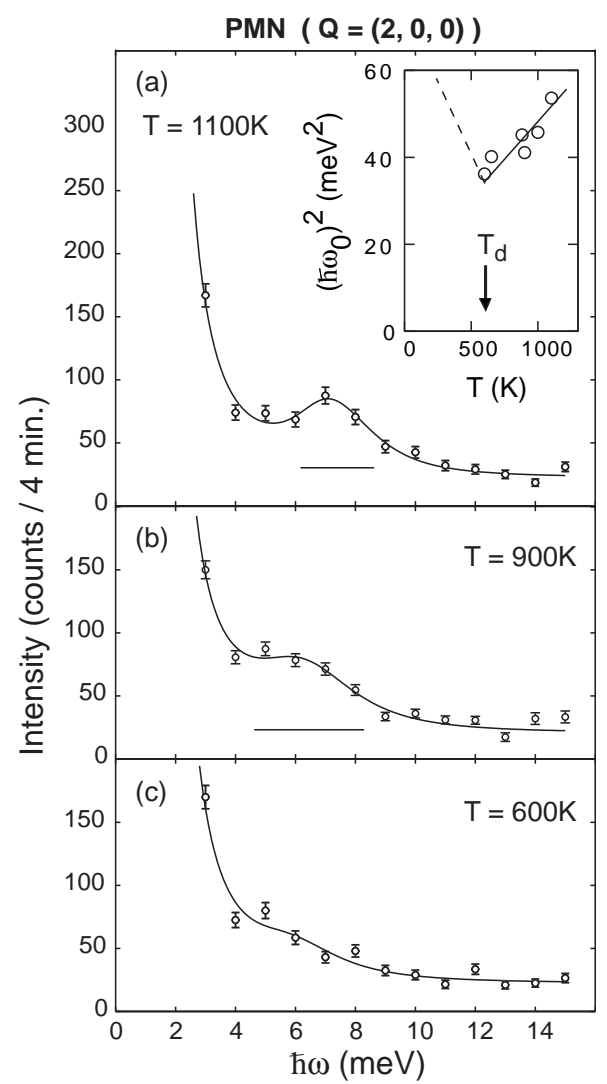

FIGURE 3. Temperature dependence of the soft TO mode in PMN. From Gehring et al. [3] ]

the zone center TO mode energy varies linearly with temperature $\left(\left(\hbar \omega_{0}^{2}\right) \sim\left(T-T_{0}\right)\right)$ as shown in the inset. This is the hallmark of a ferroelectric soft mode. While the softening of this mode is clear, the uncertainties in the data obtained from this small $0.1 \mathrm{~cm}^{3}$ sample do not permit a reliable determination of $T_{0}$, which should be close to $400 \mathrm{~K}$. [12] Data obtained on a much larger crystal $\left(0.4 \mathrm{~cm}^{3}\right)$ are discussed in a later section and are combined with the data shown in Fig. 3 (see Fig. 5).

\section{THE CONNECTION TO THE PNR}

If the PNR are in fact the result of the condensation of the soft TO mode in PMN, then the ionic displacements that characterize the diffuse scattering intensities below $T_{d}$ must be consistent with those that characterize the soft mode above $T_{d}$. In particular, since the ionic motions associated with the zone center TO vibrational mode cannot shift the center of mass of the PMN unit cell, the static displacements observed below $T_{d}$ must also satisfy this constraint. But they do not. Vakhrushev et al. measured the neutron diffuse scattering intensities near 16 reciprocal lattice points on a single crystal of PMN, and obtained the following relative ionic displacments $\delta$ (normalized such that $\left.\delta_{\mathrm{Pb}}=1\right): \delta_{\mathrm{MN}}=0.18, \delta_{\mathrm{O}(1)}=-0.738, \delta_{\mathrm{O}(2)}=-0.549$. [8] These displacements do not 

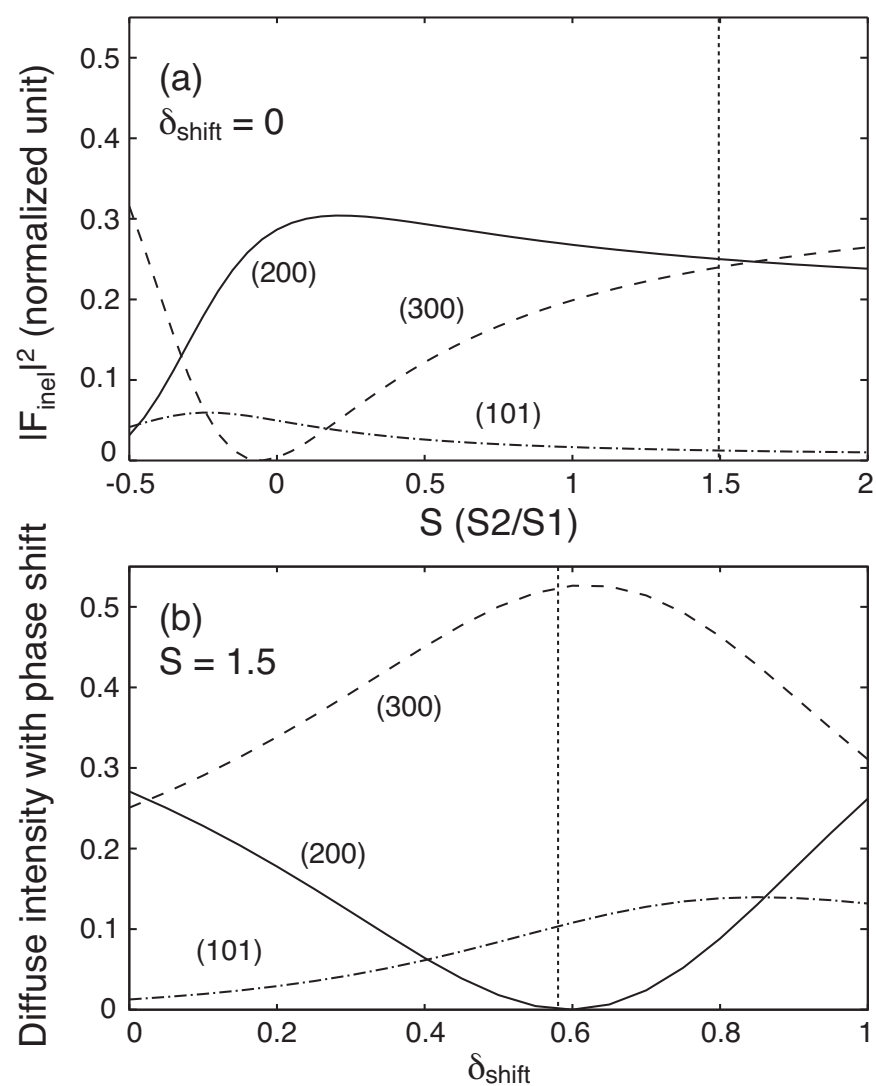

FIGURE 4. Model calculations of the inelastic and diffuse structure factors of PMN. From Hirota $e t$ al. [13]

satisfy the center-of-mass constraint. Yet if one believes that the static and dynamic ionic displacements are related to the same polar state, then one is faced with an unavoidable discrepancy that must be resolved.

This apparent contradiction was recently resolved by Hirota et al. who noticed that if a uniform shift of the proper magnitude were subtracted from each of the ionic displacments, then the remaining displacements satisfied the center-of-mass constraint. In other words, for each ion they assumed $\delta_{i}=\delta_{i}^{\mathrm{cm}}+\delta_{\text {shift }}$, where $i$ is the ionic index. Using this model, the size of the uniform shift was determined to be $\delta_{\text {shift }}=0.58$, assuming an average oxygen displacement of $\delta_{\mathrm{O}}=-0.64$. The corresponding values for the $\delta_{i}^{\mathrm{cm}}$ are: $\delta_{\mathrm{Pb}}^{\mathrm{cm}}=0.42, \delta_{\mathrm{MN}}^{\mathrm{cm}}=-0.40, \delta_{\mathrm{O}}^{\mathrm{cm}}=-1.22$. [13]

Calculations of both the inelastic and diffuse scattering structure factors were made using these values. Fig. 4 (a) shows the calculated intensities of the TO phonon mode at the (101), (200), and (300) reciprocal lattice points as a function of the ratio $S=S_{2} / S_{1}$ of the Slater $\left(S_{1}\right)$ and Last $\left(S_{2}\right)$ modes. [14] Inelastic scans measured at (200) and (300) (see Fig. 6) fix the value of $S$ close to 1.5, consistent with that for $\mathrm{PbTiO}_{3}$. Fig. 4 (b) shows the corresponding diffuse scattering intensities for this value of $S$ as a function of the single parameter $\delta_{\text {shift }}$. Agreement with experiment, which indicates extremely little diffuse scattering around (200), is obtained precisely at $\delta_{\text {shift }}=0.58$. Because of 


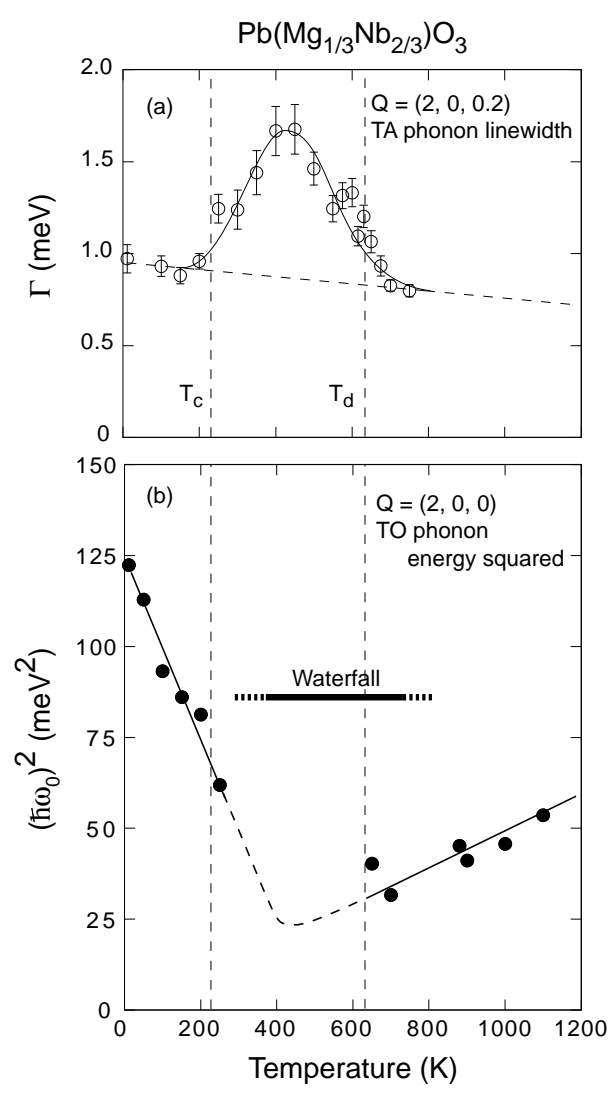

FIGURE 5. Summary of the TA linewidth and TO soft mode temperature dependence in PMN. From Wakimoto et al. [18]

the uniform shift $\delta_{\text {shift }}$, the PNR are said to result from a "phase-shifted condensed soft mode." The shift of the PNR is along their respective polar directions.

\section{FERROELECTRIC DYNAMICS IN PMN BELOW $T_{C}$}

Above $T_{d} \approx 620 \mathrm{~K}$ PMN exhibits dynamical properties characteristic of a normal ferroelectric in its cubic phase that are surprisingly similar to those observed in $\mathrm{PbTiO}_{3}$. The formation of PNR below $T_{d}$ appear to stifle the propagation of long-wavelength polar TO modes due to their finite size. The concurrent development of diffuse scattering intensity at $T_{d}$, first observed by Naberezhnov et al. [7], is consistent with the picture of PNR condensing from the soft TO mode. Yet PMN is reported to remain cubic on average at all temperatures (at least down to $5 \mathrm{~K}$ ). [15] On the other hand, it is known that a macroscopic ferroelectric phase can be induced in PMN by cooling it in a small electric field. After removal of this field, the ferroelectric state in PMN is lost via a first-order transition when heated above $T_{c}=213 \mathrm{~K}$. [16, 17] Therefore the lattice dynamical studies of PMN were extended to low temperature, placing special emphasis on the temperature region near $T_{c}$. 
Fig. 5 summarizes recent neutron inelastic scattering results obtained by Wakimoto et al. on a large $0.4 \mathrm{~cm}^{3}$ single crystal between 10 and $750 \mathrm{~K}$. [18] By far the most remarkable feature is the recovery and subsequent behavior of the soft mode where $\left(\hbar \omega_{0}\right)^{2} \sim\left(T_{0}-T\right)$ below $T_{c}$ shown in Fig. 5 (b) that is typical of many ferroelectrics in their ordered states. This implies that a phase transition from the relaxor state to a lowtemperature, short-range ordered ferroelectric state, occurs at $T_{c}$ in the absence of an applied field. But this contradicts the average cubic symmetry of PMN. However, if the length scale of the ferroelectric order were $\sim 100 \AA$, then one could plausibly resolve this contradiction since this length scale would appear as long-range order in neutron phonon measurements, but short-range order to x-rays. Indeed, regions of ferroelectric order $\sim 100 \AA$ in size have been reported by de Mathan et al. [15] Hypersonic damping [19] and Raman scattering [9] studies have also reported anomalies at or near $T_{c}$, as has a change in the topology of the PNR, [20], all of which lend support to the idea of a low-temperature ferroelectric state in PMN. It is interesting to note that in a zone center mode is observed at low $(25 \mathrm{~K})$ temperature in PZN at essentially the same energy as that found in PMN (10.5 meV). [2]

Fig. 5 (a) shows an anomalous damping of the TA mode that coincides with the overdamping of the soft mode and the appearance of the PNR at $T_{d}$. This suggests a non-uniform distortion of the lattice which then vanishes below $T_{c}$, ostensibly when the short-range ordered ferroelectric phase is established. A similar TA broadening has also been observed in PMN-20\%PT by Koo et al. [10]

\section{MODE COUPLING ANALYSIS}

The scattering cross section used to fit these inelastic data is based on the coupled-mode description first used by Harada et al. to fit asymmetric phonon lineshapes observed in $\mathrm{BaTiO}_{3}$. [21] Typical data measured in two different zones, (200) and (300), and taken above $T_{d}$ at $690 \mathrm{~K}$, are shown for PMN in Fig. 6. The coupled-mode cross section is parametrized by a coupling-constant $\lambda$, along with inelastic structure factors $F_{1}$ and $F_{2}$ for the coupled TO and TA modes, respectively. Additional parameters are the phonon energies $\hbar \omega_{1}$ and $\hbar \omega_{2}$, and the linewidths $\Gamma_{1}$ and $\Gamma_{2}$. All of these parameters were allowed to float freely between the two scans. The values for the TA and TO phonon energies were identical to within $0.1 \mathrm{meV}$ between (200) and (300), as required, with $\hbar \omega_{1}=10.8 \mathrm{meV}, \hbar \omega_{2}=4.2 \mathrm{meV}$, and represent a useful cross check of the integrity of the data. However the ratio of the dynamic structure factors $F_{1} / F_{2}$ is markedly different in each zone. At (200) the ratio is 2.3 , whereas at (300) it is 27.5 , nearly an order of magnitude different. The coupling constant $\lambda$ has nearly the same absolute value in each zone (-14.1 versus 17.6 for (200) and (300) respectively), but opposite sign. Physically this is due to a sign change in the TO structure factor $F_{1}$, not $\lambda$.

Fig. 7 conveys the manner in which the coupled-mode cross section varies with the TO mode linewidth $\Gamma_{1}$ (corresponding to an increased damping), as well as with a change in $\lambda$. This cross section gives an excellent model description, shown in panel (a), of the $q$ dependent damping observed in both PZN and PMN below $T_{d}$. Two cases are presented in panels (b) and (c) following Bullock et al. [22] Panel (b) corresponds to well-separated 

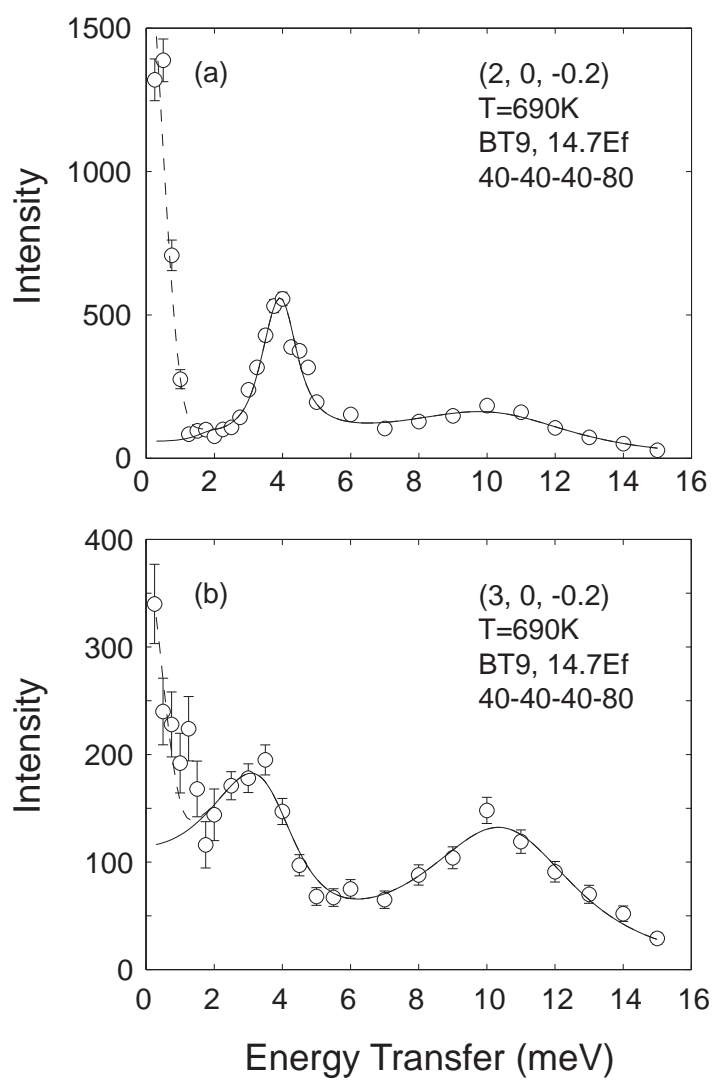

FIGURE 6. Constant $-\vec{Q}$ scans measured in PMN around the (a) (200) and (b) (300) Brillouin zones. Solid lines are fits to a coupled TA-TO mode cross section, corrected for instrumental resolution effects (data taken from Hirota et al.). [13]

TA and TO modes with energies of 5 and $10 \mathrm{meV}$, respectively, and identical linewidths of $1 \mathrm{meV}$. Panel (c) corresponds to TA and TO modes with energies of 6 and $8 \mathrm{meV}$, and substantially different linewidths of 0.5 and $4 \mathrm{meV}$, respectively, such that there is substantial spectral overlap. [22] In both cases there is a clear transfer of spectral weight from the TO mode to the TA mode when the mode coupling is turned on $(\lambda \neq 0)$.

\section{CONCLUSIONS}

We have given a brief overview of the lattice dynamics of PMN and PZN. In PMN a well-defined soft mode is observed at $1100 \mathrm{~K}$ that becomes increasingly damped upon cooling, and eventually becomes overdamped at $T_{d}$. The overdamping of the soft mode and the appearance of the diffuse scattering both at $T_{d}$ strongly point to a direct connection between the soft mode and the PNR. The resolution of the discrepancy between the static and dynamic structure factors using the "phase-shifted condensed soft mode" model of Hirota et al. provides a convincing framework in which this connection can be understood. The damping of the soft mode that is present at temperatures above 
Mode-coupling function

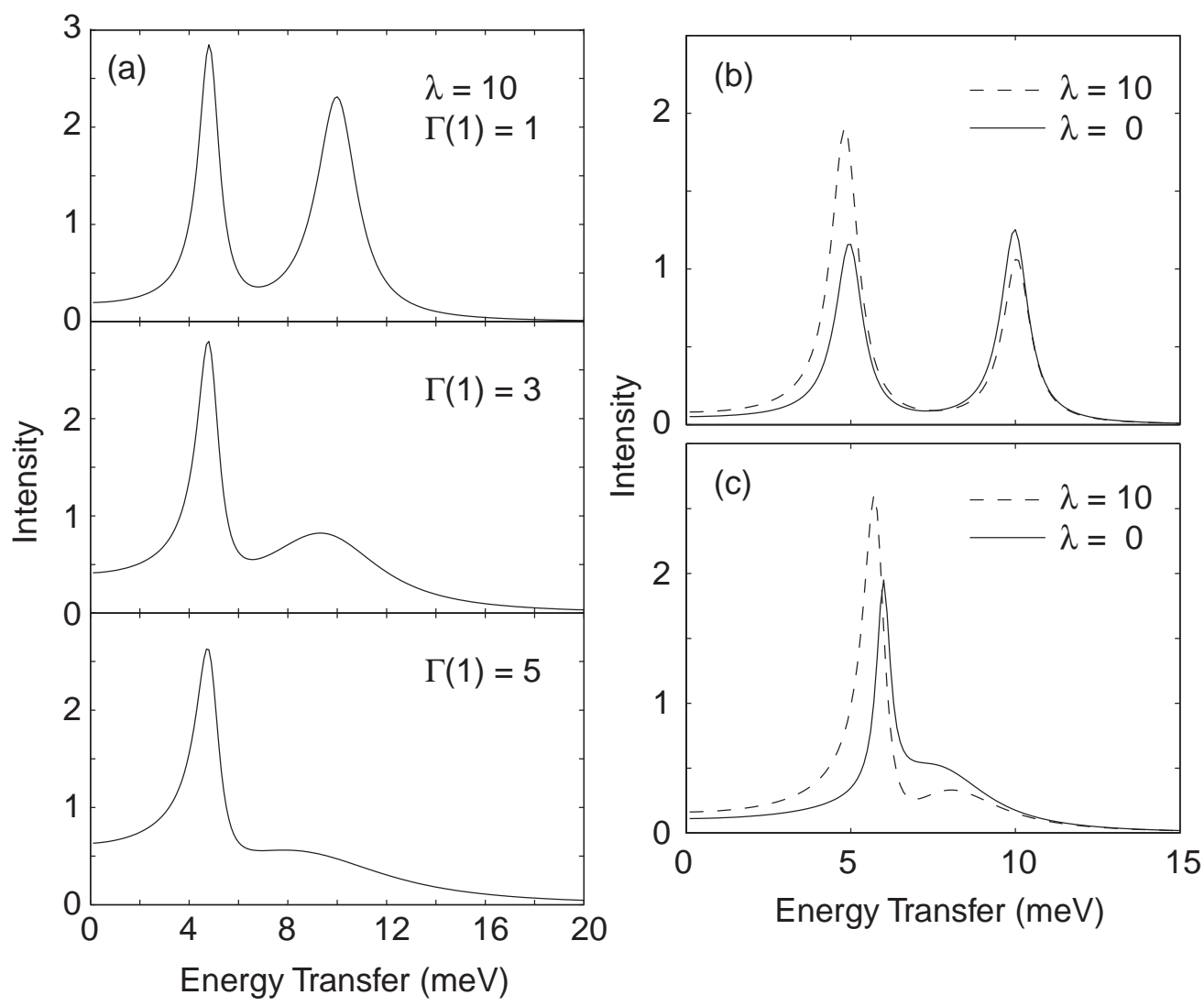

FIGURE 7. (a) Variation of coupled-mode cross section with TO linewidth $\Gamma_{1}$. Comparison of the coupled-mode cross section with and without coupling when modes (b) are well-separated, and (c) have significant spectral overlap. Parameters used are identical to those in Fig. 7 by Bullock et al. [22]

$T_{d}$ is believed to be a result of the fact that the PNR exist as dynamical entities at higher temperature.

The soft mode dynamics of PMN below $T_{c}$ are characteristic of an ordered ferroelectric phase, and stand in contrast to the average cubic structure reported for PMN. The assumption of short-range order $(\sim 100 \AA)$ can be used to reconcile these two sets of experimental observations. At all temperatures, a coupled-mode cross section, properly convolved with the instrumental resolution function, provides an excellent description of the neutron inelastic data.

A very different interpretation of the soft mode dynamics in PMN has recently been proposed by Vakhrushev and Shapiro [23] in which the existence of a soft "quasi-optic" (QO) mode, distinct from the zone center TO mode, is claimed to explain the observed Curie-Weiss behavior of the dielectric susceptibility above $T_{d}$. The QO-mode is derived from a mode-coupling analysis of neutron inelastic lineshapes measured between 490 and $880 \mathrm{~K}$, and is attributed to the intrinsic chemical disorder of PMN. More studies of PMN at higher resolution are clearly needed to reconcile these different points of view. 


\section{ACKNOWLEDGMENTS}

We thank A. A. Bokov, K. Hirota, V. Kiryukhin, T. -Y. Koo, K. Ohwada, S. M. Shapiro, C. Stock, N. Takesue, S. B. Vakhrushev, and H. You for stimulating discussions. Financial support from the U. S. DOE under contract No. DE-AC02-98CH10886, and the Office of Naval Research under Grant No. N00014-99-1-0738, is acknowledged. Work at the University of Toronto is part of the Canadian Institute for Advanced Research and is supported by the Natural Science and Engineering Research Council of Canada. We acknowledge the support of the NIST Center for Neutron Research, the U. S. Department of Commerce, for providing some of the neutron facilities used in the present work.

\section{REFERENCES}

1. P. M. Gehring, S.-E. Park, and G. Shirane, Phys. Rev. Lett. 84, 5216 (2000).

2. P. M. Gehring, S.-E. Park, and G. Shirane, Phys. Rev. B 63, 224109 (2000).

3. P. M. Gehring, S. Wakimoto, Z.-G. Ye, and G. Shirane, Phys. Rev. Lett. 87, 277601 (2001).

4. See review article, Z.-G. Ye, Key Engineering Materials Vols. 155-156, 81 (1998).

5. G. Burns and F. H. Dacol, Solid State Commun. 48, 853 (1983).

6. G. Shirane, J. D. Axe, and J. Harada, Phys. Rev. B 2, 155 (1970).

7. A. Naberezhnov, S. Vakhrushev, B. Doner, D. Strauch, and H. Moudden Eur. Phys. J. B 11, 13 (1999)

8. S. B. Vakhrushev, A. A. Naberezhnov, N. M. Okuneva, and B. N. Savenko, Phys. Solid State 371993 (1995).

9. I. G. Siny, S. G. Lushnikov, R. S. Katiyar, and E. A. Rogacheva, Phys. Rev. B 56, 7962 (1997).

10. T.-Y. Koo, P. M. Gehring, G. Shirane, V. Kiryukhin, G. Lee, and S.-W. Cheong, cond-mat/0110531, accepted by Phys. Rev. B (in press).

11. D. La-Orauttapong, B. Noheda, Z.-G. Ye, P. M. Gehring, J. Toulouse, D. E. Cox, and G. Shirane, cond-mat/0108264, accepted by Phys. Rev. B (in press).

12. D. Viehland, S. J. Lang, L. E. Cross, and M. Wuttig, Phys. Rev. B 46, 8003 (1992).

13. K. Hirota, Z.-G. Ye, S. Wakimoto, P. M. Gehring, and G. Shirane, cond-mat/0109386(Phys. Rev. B in press).

14. J. Harada, J. D. Axe, and G. Shirane, Acta Cryst. A 26, 608 (1970).

15. N de Mathan, E. Husson, G. Calvarin, J. R. Gavarri, A. W. Huwat, and A. Morell, J. Phys. Condens. Matter 3, 8159 (1991); P. Bonneau, P. Garnier, G. Calvarin, E. Husson, J. R. Gavarri, A. W. Hewat, and A. Morell, J. Solid State Chem. 91, 350 (1991).

16. Z.-G. Ye and H. Schmid, Ferroelectrics 145, 83 (1993).

17. G. Calvarin, E. Husson, and Z.-G. Ye, Ferroelectrics 165, 349 (1995).

18. S. Wakimoto, C. Stock, R. J. Birgeneau, Z.-G. Ye, W. Chen, W. J. L. Buyers, P. M. Gehring, and G. Shirane, cond-mat/0112366, submitted to Phys. Rev. B.

19. C.-S. Tu, V. Hugo Schmidt, and I. G. Siny, J. Appl. Phys. 78, 5665 (1995).

20. S. Vakhrushev, A. Naberezhnov, S. K. Sinha, Y. P. Feng, and T. Egami, J. Phys. Chem. Solids 57, 1517 (1996).

21. J. Harada, J. D. Axe, and G. Shirane, Phys. Rev. B 4, 155 (1971).

22. M. Bullock, J. Zaretsky, C. Stassis, A. Goldman, P. Canfield, Z. Honda, G. Shirane, and S. M. Shapiro, Phys. Rev. B 57, 7916 (1998).

23. S. B. Vakhrushev and S. M. Shapiro, cond-mat/0203103. 